\title{
Mitochondria association to Calcium Release Units is controlled by age and muscle activity
}

\author{
Feliciano Protasi
}

CeSI - Center for Research on Ageing \& DNICS - Dept. of Neuroscience,
Imaging, and Clinical Sciences, Univ. G. d'Annunzio of Chieti, I-66013 Italy.

Background. At the most basic level, skeletal muscle contraction requires $\mathrm{Ca}^{2+}$ and ATP and, thus, is under direct control of two important intracellular organelles: $\mathrm{Ca}^{2+}$ release units (CRUs) - specialized intracellular junctions, also named triads, which are involved in excitation-contraction (EC) coupling - and mitochondria, the organelles deputed to produce the energy required for most cellular functions (i.e. aerobic ATP production). It is now becoming clear that: a) CRUs and mitochondria interact functionally and structurally, as entry of $\mathrm{Ca}^{2+}$ into the mitochondrial matrix is required to stimulate the respiratory chain, and increase production of ATP (Fig. 1) (Sembrowich et al. $1985^{1}$; Brookes et al. $2004^{2}$; Rossi et al. 2009) ${ }^{3}$; b) we recently discovered that, in adult skeletal muscle fibers, mitochondria and CRUs are placed in close proximity to each other (Fig. 2) and structurally linked by small strands called tethers (Fig. 3) (Boncompagni et al. 2009) ${ }^{4}$.

Scientific hypothesis of the study. Miss-function of mitochondria and functional/structural changes affecting the EC coupling apparatus have been both proposed to contribute to the age-related decline of skeletal muscle performance (Delbono et al. $19955^{5}$; Boncompagni et al. $2006{ }^{6}$ ). In this study, we tested the following hypothesis: muscle activity improves/maintains the correct association between CRUs and mitochondria, which is challenged by ageing and inactivity.

Experimental Plan. We have studied the morphology, frequency, and sarcomericlocalization of both CRUs and mitochondria using light, confocal, and electron microscopy (EM) in: a) Extensor Digitorum Longus (EDL) muscles from adult (3-12 months of age) and ageing ( $\geq 24$ months of age) wild type (WT) mice; and b) in human biopsies from sedentary elderly subjects ( $70 \pm 5$ years) and age matched sportmen (69 \pm 4 years of age) to determine how EC coupling and mitochondrial apparatuses are affected by age and exercise.

Results $\boldsymbol{A}$. Studies in mice revealed that: a) the number of CRUs $/ 100 \mu \mathrm{m}^{2}$ (measured in longitudinal EM sections) in aging mice decreases significantly compared to adult mice: $87.4 \pm 30.3$ vs. $74.1 \pm 25.1$, respectively $(p<0.01)$; b) the number of mitochondria-profiles $/ 100 \mu \mathrm{m}^{2}$ also decreases with age: $52.9 \pm 23.5 \mathrm{vs}$. $42.8 \pm 21.0$, respectively $(\mathrm{p}<0.01) ; \mathrm{c})$ in ageing fibers mitochondria are more frequently found at the $A$ band of the sarcomere $(5.9 \pm 3.3$ vs. $1.5 \pm 5.3)$, i.e. away from CRUs. The miss-placement of mitochondria is likely the results of the decreased frequency of tethers: in ageing fibers their number decreased with age from $14 / 100 \mu \mathrm{m}^{2}$ in adult vs. $6 / 100 \mu \mathrm{m}^{2}$ in ageing mice. The above changes taken together caused a significant decrease in the number CRUs-mitochondria couples/100 $\mu \mathrm{m}^{2}$ : $37.4 \pm 17.4$ vs. $27.0 \pm 15.8$ (a decrease of $\sim 27 \%$ ). This reduction of $\mathrm{CRU} /$ mitochondria couples may significantly contribute to the decrease of specific force and endurance of skeletal muscle associated to ageing. A manuscript containing these data was recently submitted for publication (Pietrangelo \& D'Incecco et al. submitted).

In order to determine if the structural changes described above are caused by ageing itself or if inactivity plays also a central role in the progressive decay of EC coupling and mitochondrial apparatuses, we also studied mice that had access to running wheels for the second part of their lives (from 1 to 2 years of age). Results collected from these (unpublished) findings indicated that exercise improves number of mitochondria (a), their position with respect to sarcomeric striation (b), and their association to CRUs (c): a) number of mitochondria: $49.3 \pm 19.8^{*} /$ $100 \mu \mathrm{m}^{2}$; b) number of mitochondria at the $A$ band: $2.0 \pm 4.2^{*} / 100 \mu \mathrm{m}^{2}$; number of CRUs-mitochondria couples: $35.0 \pm 16.8^{*} / 100 \mu \mathrm{m}^{2}$. Please compare these data 
with results from sedentary mice reported above (differences were all highly significant: ${ }^{*} p<0.01$ ).

Results $\boldsymbol{B}$. Studies in human Vastus Lateralis biopsies from sedentary elderly subjects confirmed general findings collected in mice (i.e. decrease in frequency of both CRUs and mitochondria and partial miss-placement of mitochondria). In human studies (see Figure 4), we compared samples from two groups of elderly individuals (all males): sedentary subjects ( $70 \pm 5$ years) or sportmen (69 \pm 4 years of age), i.e. individuals who regularly exercised in the last several years of their lives. These studies revealed that both CRUs and mitochondria increase with exercise, mitochondria more than CRUs. Number of CRUs / $100 \mu \mathrm{m}^{2}: 20.3 \pm 10.0$ in sedentary vs. $21.6 \pm 10.8$ in sportsmen; number of mitochondria / $100 \mu \mathrm{m}^{2}: 37.1$ \pm 18.3 in sedentary vs. $52.0 \pm 21.3$ in sportsmen. The combined increase of both CRUs and mitochondria resulted in largely increased frequency of $\mathrm{CRU} /$ mitochondria pairs $/ 100 \mu \mathrm{m}^{2}: 5.9 \pm 5.5$ in sedentary vs. $11.1 \pm 8.3$ in sportsmen. These studies have been recently published in a larger study including, beside the just described EM analysis, also functional and histological data (Zampieri et al. 2015). ${ }^{7}$

Discussion and Perspectives. The results collected in our studies suggest that structure/organization of both EC coupling and mitochondrial apparatus: a) is affected by age; b) is better preserved by exercise. In conclusion, the dramatic age-related decay affecting mitochondrial and EC coupling apparatuses in skeletal muscle of mice and humans are, at least in part, caused by inactivity due to changes in life style.

Key words: Ageing, EC coupling, exercise, skeletal muscle.

Eur J Transl Myol - Basic Appl Myol 2015; 25 (4): 257-262

Slide 1. Good morning to everybody. It is pleasure for me to be here today, once again in Vienna and to report about this project that we are conducting in collaboration with Prof. Helmut Kern and with our colleagues in Padova, Prof. Ugo Carraro and Dr. Sandra Zampieri.

In this study, we are investigating the effects of ageing and exercise on the association between two organelles which are crucial for muscle function: calcium release units (CRUs) and mitochondria. ${ }^{2}$

\section{Slide 2.}

Excitation Contraction (EC) Coupling, the mechanism that triggers release of $\mathrm{Ca}^{2+}$ from the Sarcoplasmic Reticulum (SR) in response to an action potential propagating in the transverse $(\mathrm{T})$ tubule - occurs in CRUs, the specialized intracellular

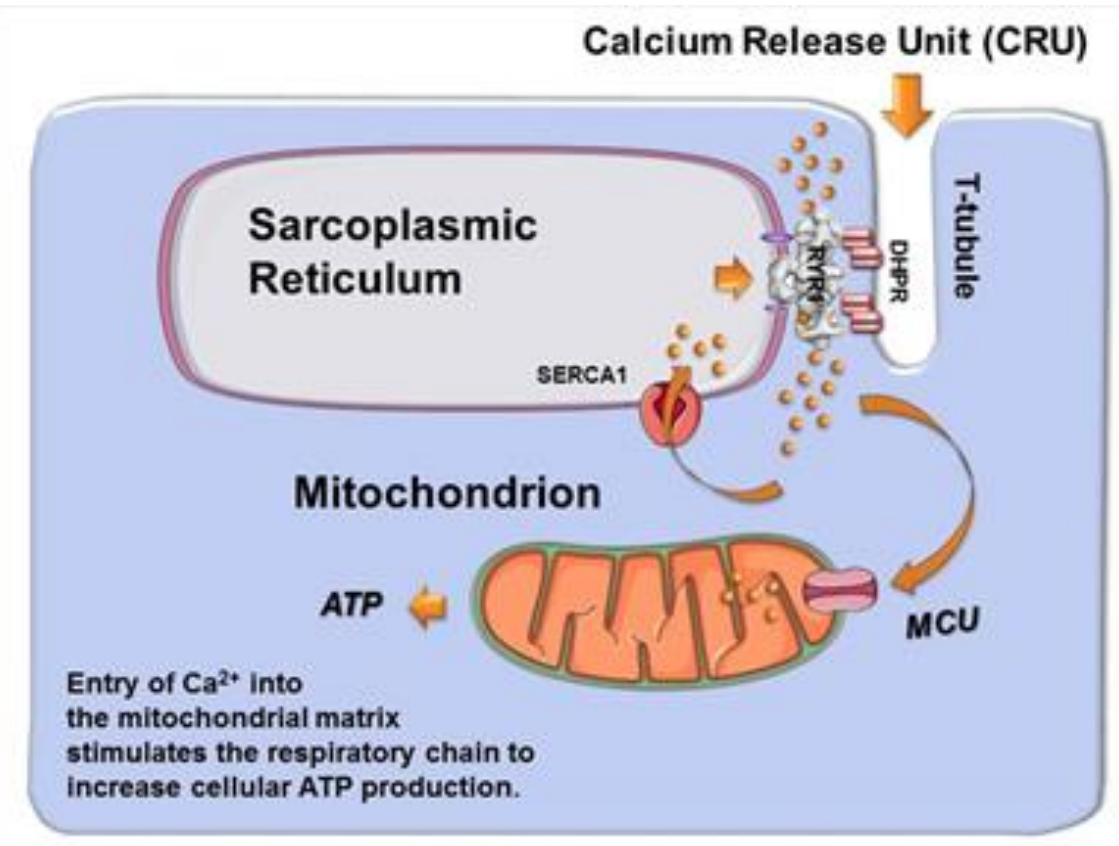

Fig 1. $\mathrm{Ca}^{2+}$ signaling between CRUs and mitochondria. A small amount of $\mathrm{Ca}^{2+}$ released by CRUs during EC coupling enters mitochondria through a protein known as mitochondrial $\mathrm{Ca}^{2+}$ uniporter (MCU) (De Stefani et al., 2011): ${ }^{8}$ this entry of $\mathrm{Ca}^{2+}$ into the mitochondrial matrix stimulates the aerobic metabolism to increase cellular ATP production. 


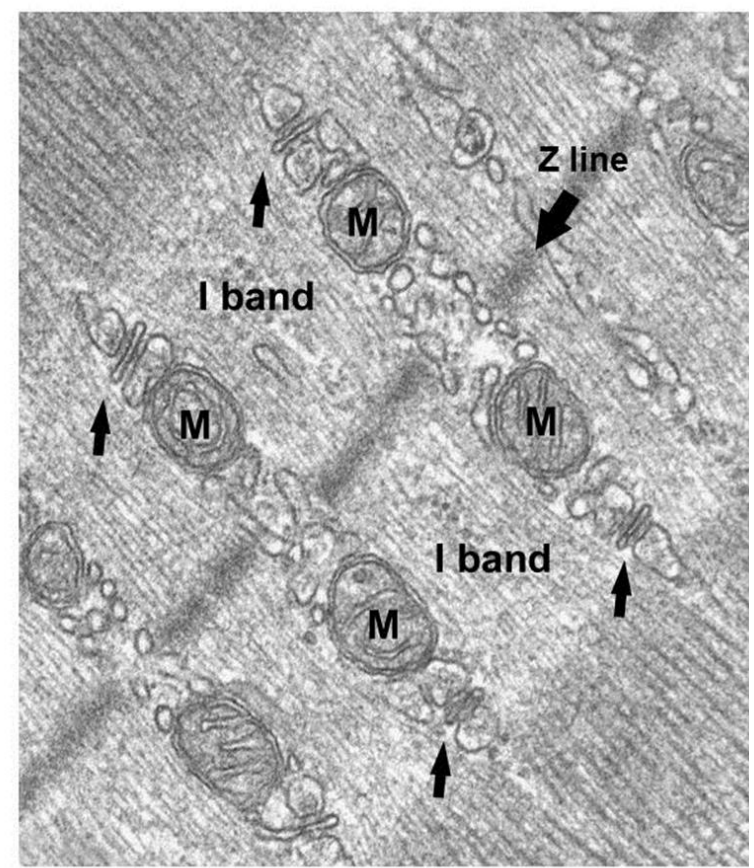

Fig 2. Disposition of CRUs (or triads) and mitochondria in skeletal muscle fibers. In this electron-micrograph, CRUs (also called triads because formed by three elements) are pointed by arrows and specifically positioned at the edges of the I band of relaxed sarcomeres. Mitochondria (marked with $\mathrm{M}$ ) are placed at the I band next to triads, always on the side closer to the $Z$ line: in this position these organelles are strategically disposed to uptake $\mathrm{Ca}^{2+}$ released from CRUs during EC coupling (see also Fig. 1).

junctions that in skeletal muscle are formed by 3 elements, two SR terminal cisternae flanking a central T- tubule. For this reason CRUs are also called triads. In this electron micrograph you can see the structure of a triad and intracellular positioning of triads with respect to the striation of sarcomeres.

Slide 3.

As many of you already know, our laboratory has special interest in: a) EC coupling, which is controlled by a macromolecular complex including among other proteins - ryanodine receptors (RYRs), the $\mathrm{SR} \mathrm{Ca}{ }^{2+}$ release channels, and dihydropyridine receptors (DHPRs), the T- tubule voltage sensors; and b) in electron microscopy (EM), a technique that allows us to study intracellular organelles in muscle fibers at high magnification/resolution.

\section{Slide 4.}

In 2004 the group of Tullio Pozzan in Padova published a paper showing how mitochondria, the organelles deputed to aerobic ATP production, in mammalian skeletal muscle take up $\mathrm{Ca}^{2+}$ very quickly during contraction induced by nerve stimulation.

\section{Slide 5.}

Entry of $\mathrm{Ca}^{2+}$ into mitochondria is a phenomenon known since many years ago and occurs through the mitochondrial $\mathrm{Ca}^{2+}$ uniporter (MCU), a protein that was unknown for many years, but that has been finally identified by two independent groups, one of them being the group of Rosario Rizzuto, again in Padova. Functionally, entry of $\mathrm{Ca}^{2+}$ into the mitochondrial matrix is metabolically important as it stimulates the respiratory chain and increases cellular ATP production during muscle contraction. However, in this picture there is an apparent paradox: indeed, when mitochondria are isolated from the tissue that contains them, $\mathrm{Ca}^{2+}$ concentration required to activate mitochondrial $\mathrm{Ca}^{2+}$ uptake is much higher (about $30 \mu \mathrm{M}$ ) than the concentration reached during $\mathrm{Ca}^{2+}$ transients in-situ (only about $1 \mu \mathrm{M}$ ). This discrepancy has lead the physiologists to develop the hypothesis of local $\mathrm{Ca}^{2+}$ microdomains, which proposes that mitochondria to take up $\mathrm{Ca}^{2+}$ efficiently - must be placed in close proximity to site of $\mathrm{Ca}^{2+}$ release.

\section{Slide 6.}

To give our contribute in solving this fundamental question we have started to become interested in the interaction between CRUs (or triads) and mitochondria in skeletal muscle fibers. In this EM tomography you see how the two organelles are often in close proximity to one another.

\section{Slide 7 and 8.}

In 2009 we published a paper in Molecular Biology of the Cell in which we studied the intracellular positioning of mitochondria and CRUs in fast twitch skeletal muscle from mice during post-natal maturation. ${ }^{4}$ Whereas in developing fibers the proximity of mitochondria to CRUs is only partial (as many mitochondria are positioned longitudinally), in adult fibers mitochondria are specifically placed in I bands next to triads, which in turn are located at the edges of I bands, i.e. at the transition I and A bands when the sarcomeres are relaxed.

\section{Slide 9.}

In this study we also reported that under high magnification, electron dense small strands appear to tether, or bridge, individual mitochondria to parajunctional regions of the SR terminal cisternae.

\section{Slide 10.}

This rotating cartoon - generated by Simona Boncompagni in collaboration with a group at Mario Negri Sud in Lanciano, Italy - is indeed the 3dimentional reconstruction (obtained from the EM tomography you see over here) of a CRU associated to a mitochondrion. The two organelles the triad in yellow and white, and the mitochondrion in green - in adult fast-twitch fibers are indeed closely associated to one another forming functional couples. In red you see the tethers that you just saw in the EM pictures in the previous slide. Which is the possible role of tethers in skeletal muscle fibers?

\section{Slide 11.}

Mitochondria are known to be quite mobile in the cytoplasm of various type of cells. In this movie (which is 20 minutes long) you see mitochondria labelled in green forming a network inside this Human Embryonic Kidney (or HEK) cell: you can easily appreciate how this network moves with time. 


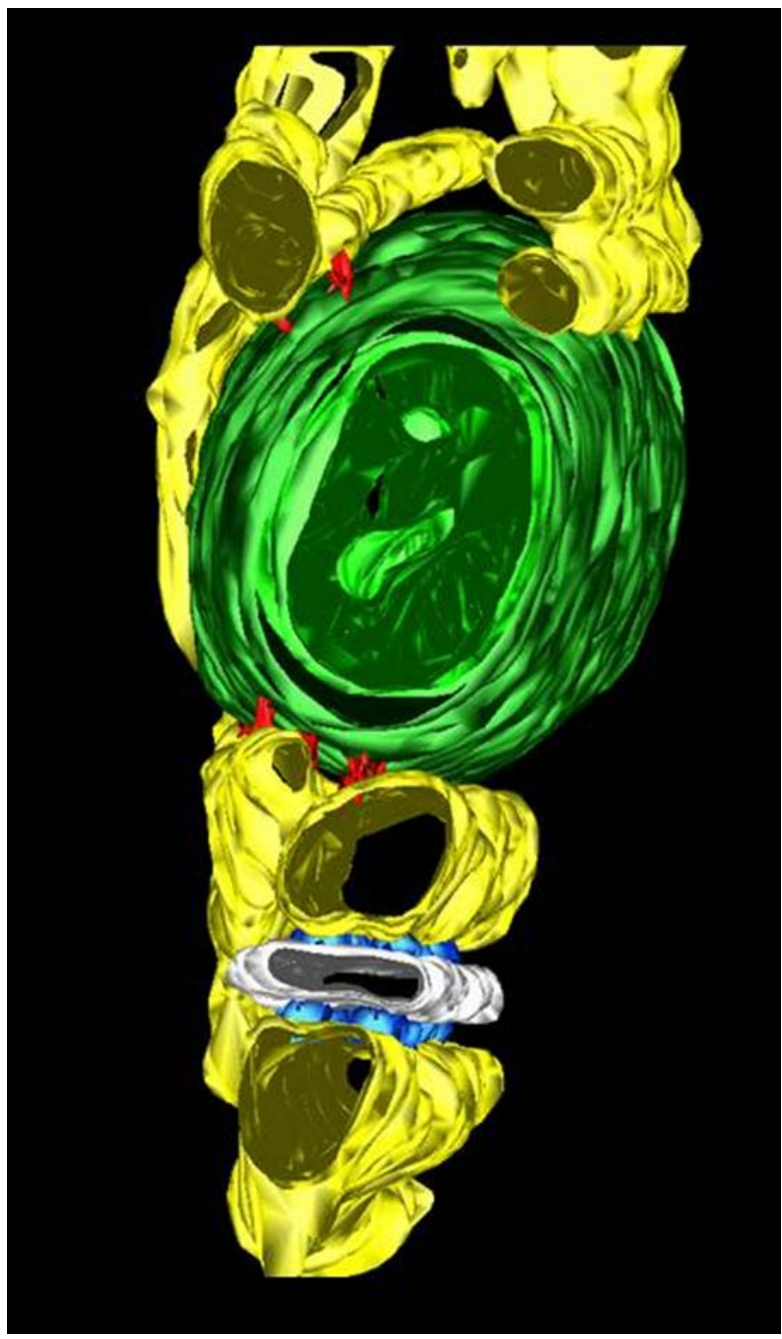

Fig 3. 3D reconstruction of a CRU with an associated mitochondrion. In adult skeletal muscle fibers, CRUs (in white, cyan and yellow) and mitochondria (in green) are placed in close proximity to each other (see also Fig. 2) and structurally linked to one another by small strands called tethers (in red). Colour legend: yellow, SR; white, T-tubule; cyan, RYRs; green, mitochondrion; red, tethers. See Rossi et al. 2009 and Boncompagni et al. 2009 for more details. ${ }^{3,4}$

\section{Slide 12.}

However, when looking at a movie of the same length (again 20 minutes) taken form a skeletal muscle fibers we see immediately how these mitochondria here do not move, as in the previous slide For this reason we believe that tethers are likely important for holding mitochondria in a specific sub-cellular position in muscle fibers (i.e. next to sources of $\mathrm{Ca}^{2+}$ release).

Slide 13.

Experiments in confocal microscopy using fluorescent dyes that reveal the polarization state of mitochondria indicates that position of mitochondria next to CRUs affects their functional state, underlying once more how tethers may play an important function.

\section{Slide 14}

This slide summarizes the first part of the talk: triads are placed in a specific position with respect to sarcomeres, with mitochondria blocked next to them. We believe that this spatial disposition is very important for SR-mitochondria $\mathrm{Ca}^{2+}$ signaling (and likely for proper metabolic efficiency of muscle fibers). As an indirect evidence for this hypothesis, we are getting more and more aware (thanks to different projects running in the lab) that this precise organization is challenged in several physiopathological conditions such as ageing, in several myopathies and also in denervation.

\section{Slide 15.}

The Scientific Hypothesis of the Project that I will present to you in the next slides is that muscle activity maintains/improves the correct association between CRUs and mitochondria, which - we hypothesized - is challenged by ageing and inactivity.

To prove our hypothesis we are currently working on 3 different groups of samples:

a. In collaboration with Helmut Kern here in Vienna, and Sandra Zampieri and Ugo Carraro in Padova in a project involving also Profs. Antonio Musarò and Marco Sandri - we are analyzing muscle biopsies from Vastus Lateralis of young adults, sedentary seniors and age-matched sportmen, who had exercised regularly at least in the second part of their life (mostly bikers, skiers and/or training in the gym).

b. Extensor Digitorum Longus (EDL) muscles from controls, ageing and age-matched mice which had access to training wheels for 1 year (starting at 1 year up to 2 years of age).

c. EDL muscles from rats: controls, denervated for 15 days by nerve crash, and denervated + reinnervated for $15+15$ days.

\section{Slide 16.}

Let's start to describe the results obtained from human biopsies: Using electron microscopy (EM) we analyzed frequency (expressed as number / area of section), morphology and orientation of CRUs and mitochondria. Today - due to time constrain - I will only show data for the mitochondrial analysis, which gave the more striking results. We evaluated first mitochondrial volume and number, then positioning inside the fiber (counting how many times we see a mitochondrion at the $A$ band as here), and finally how many times mitochondria and triads are seen one next to the other, as you in panel B, to form a functional couple. With age, volume, number and association of mitochondria with CRUs decrease dramatically, whereas the only increasing parameter is the percentage of organelles found at the A band, indicative of mitochondria loosing correct I band positioning and association with triads. In sportmen though, these age-related changes are prevented: number and volume of mitochondria are well maintained, with a lower percentage of them located at the A band. These changes - combined with a small increase in the number of triads (which I did not show you) result in a number of mitochondria / CRU pairs 
Ludwig Boltzmann Institute of Electrical Stimulation and Physical Rehabilitation, Vienna, Austria

Report presented to the Interreg IVa Final Meeting November 8th 2014, Vienna, Austria

Presenter: Feliciano Protasi, Professor of Physiology, Chieti University, Italy

Eur J Transl Myol - Basic Appl Myol 2015; 25 (4): 257-262

which is 3 times higher in seniors practicing sports than in age-matched sedentary individuals.

Slide 17.

These studies (including the figure you see here) have been recently published in a larger study including, beside the EM analysis described in the previous slide, also functional and histological data collected in Vienna and in Padua (Zampieri et al. 2015). ${ }^{7}$

\section{Slide 18.}

We have also started a parallel study in ageingexercising mice: these mice had access to training wheels for 12 months from 1 to 2 years of age, mimicking those human subjects that I presented in the previous slides, which have had exercised regularly for at least the second part of their lives.

As you see here, average body weight - which increases in sedentary animals - was reduced in exercising mice. We have not yet data, though, on ratio between fat and lean mass.

On the other hand, grip strength force - which declines in sedentary animals - is higher in those mice that had the possibility of exercising on the running wheels.

\section{Slide 19 and 20.}

Qualitative analysis by both confocal and electron microscopy shows how mitochondria tend to loose proper position in ageing muscle fibers: (slide 20) this misplacement of mitochondria is accompanied by a significant decrease in the number of tethers, again supporting the idea that tethers may play a role in keeping mitochondria next to sites of $\mathrm{Ca}^{2+}$ release.

Slide 21.

In EDL of mice we then quantified also frequency and disposition of mitochondria, exactly as we did in the human biopsies.

Data show how mitochondrial number and positioning - which is negatively affected by ageing is significantly improved by running wheel exercise see red numbers - with a final $30 \%$ increase in the frequency of mitochondria-CRU pairs, a result comparable to that obtained in human biopsies.

\section{Slide 22.}

We are also starting to analyze EDL from rats after denervation by nerve crash, before and after giving the nerve time to re-innervate the muscle.

In this model of inactivity (that simply involves a period of muscle paralysis followed by rescue of movements due to re-innervation) mitochondrial number and volume are not significantly affected, but position at the I band and their association with CRUs are.

As you see by the numbers reported in this Table, denervation indeed results in mitochondria partial misplacement and decreased association to CRUs, whereas these parameters are rescued when enough time was given to the nerve to re-innervate the muscles.

\section{Slide 23.}

In this final cartoon we attempted to model changes caused by ageing: CRUs (in white and yellow) and

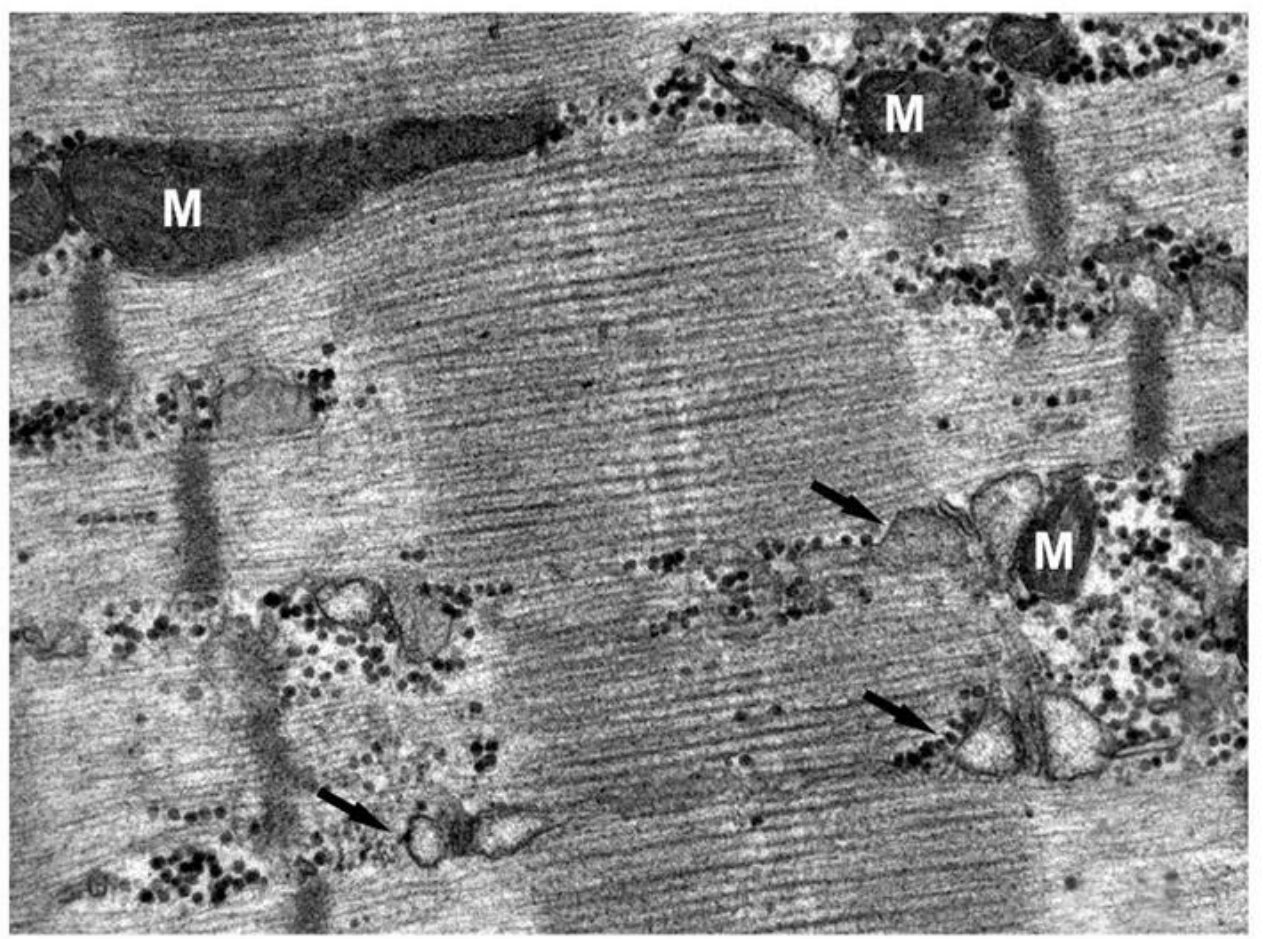

Fig 4 Representative electron micrograph taken from a human biopsy from a sedentary individual. The quantitative analysis carried in this study indicates that association between CRUs (pointed by arrows) and mitochondria (marked with $M$ ) is negatively affected by ageing, whereas exercise either maintains or rescues the association between the two organelles. Functional implication of these results is potentially large: indeed, efficient $\mathrm{Ca}^{2+}$ uptake into mitochondria likely depends on proximity of these organelles to sites of $\mathrm{Ca}^{2+}$ release (see Figs. 1-3). 
mitochondria (in green) decrease in frequency and lose proper orientation and association with increasing age. We believe that the decreased frequency of mitochondria-CRU couples contributes to the decreased efficiency of ageing muscle to produce energy and, hence, would results in reduced endurance and fatigability. Importantly though, we have also shown that exercise during ageing can almost completely rescue these changes supporting the idea that inactivity - not age per-se - is the main cause of decay, a finding which is supported also by preliminary findings in denervated EDL.

\section{Slide 24-26.}

Lately we have also started to analyze muscle biopsies from elderly people who trained with two different protocols (functional electrical stimulation, or FES, or leg-press) for 9 weeks. ${ }^{9}$

Unfortunately, we did not detect any difference in these samples with our experimental approach. However, from data collected by Profs. Antonio Musarò in Rome and Marco Sandri in Padova we do know that at the molecular level the two training protocols are effective.

Why these results in our study? The training was only 9 weeks and I think that it may take longer time to see changes at the ultrastructural level.

We are already working to get samples from elderlies who trained for longer times.

\section{Slide 27.}

To conclude, I would like to thank all the people that contributed to produce the data presented in theses slides at our institution, and at the Universities of Vienna and Padova and, finally, all of you for being here and for your attention.

\section{Abbreviations used:}

ATP, adenosine tri-phosphate; $\mathrm{Ca}^{2+}$, calcium ion; $\mathrm{CRU}, \mathrm{Ca}^{2+}$ release unit; DHPR, dihydropyridine receptor; EC coupling, excitation-contraction coupling; EDL, extensor digitorum longus; EM, electron microscopy; MCU, mitochondrial $\mathrm{Ca}^{2+}$ uniporter; RYR, ryanodine receptor; SR, sarcoplasmic reticulum; T-tubule, transverse-tubule; WT, wild type.

\section{Address for correspondence:}

Feliciano Protasi, Professor, CeSI - Center for Research on Ageing, Via Luigi Polacchi, Università G. d'Annunzio of Chieti, Italy.
E-mail: fprotasi@unich.it Phone: + 390871541423 Fax: + 390871541422

\section{References}

1. Sembrowich WL, Quintinskie JJ, Li G. Calcium uptake in mitochondria from different skeletal muscle types. J Appl Physiol 1985;59:137-41.

2. Brookes PS. Mitochondrial nitric oxide synthase. Mitochondrion 2004;3:187-204.

3. Rossi AE, Boncompagni S, Dirksen RT. Sarcoplasmic reticulum-mitochondrial symbiosis: bidirectional signaling in skeletal muscle. Exerc Sport Sci Rev. 2009 Jan;37(1):29-35. doi: 10.1097/JES.0b013e3181911fa4. Review.

4. Boncompagni S, Rossi AE, Micaroni M, et al. Mitochondria are linked to calcium stores in striated muscle by developmentally regulated tethering structures. Mol Biol Cell 2009;20:105867. doi: 10.1091/mbc.E08-07-0783. Epub 2008 Nov 26.

5. Delbono O, Chu A. $\mathrm{Ca}^{2+}$ release channels in rat denervated skeletal muscles. Exp Physiol. 1995;80:561-74.

6. Boncompagni S, d'Amelio L, Fulle $S$, et al. Progressive disorganization of the excitationcontraction coupling apparatus in aging human skeletal muscle as revealed by electron microscopy: a possible role in the decline of muscle performance. J Gerontol A Biol Sci Med Sci 2006;61:995-1008.

7. Zampieri S, Pietrangelo L, Loefler S, et al. Lifelong Physical Exercise Delays AgeAssociated Skeletal Muscle Decline. J Gerontol A Biol Sci Med Sci 2015;70:163-3.

8. De Stefani D, Raffaello A, Teardo E, et al. A forty-kilodalton protein of the inner membrane is the mitochondrial calcium uniporter. Nature 2011 Jun 19;476(7360):336-40. doi: 10.1038 /nature10230.

9. Kern $H$, Barberi L, Löfler $S$, et al. Electrical stimulation counteracts muscle decline in seniors. Front Aging Neurosci. 2014 Jul 24;6:189. doi: 10.3389/fnagi.2014.00189. eCollection 2014. 